JOURNAL OF

SYNCHROTRON

RADIATION

ISSN 1600-5775

Received 17 October 2018

Accepted 11 March 2019

Edited by M. Yabashi, RIKEN SPring-8 Center, Japan

1 This article will form part of a virtual special issue on X-ray free-electron lasers.

Keywords: FLASH; intense $\mathrm{THz}$; THz diagnostic; electro-optic; FTIR.

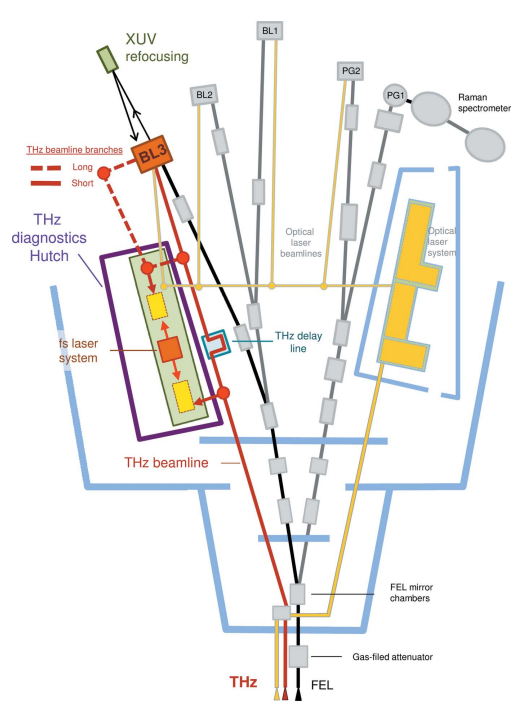

OPEN $\odot$ ACCESS

\section{Photon diagnostics at the FLASH THz beamline ${ }^{1}$}

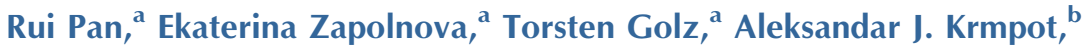 \\ Mihailo D. Rabasovic, ${ }^{\mathrm{b}}$ Jovana Petrovic, ${ }^{\mathrm{c}, \mathrm{d}}$ Vivek Asgekar, ${ }^{\mathrm{e}}$ Bart Faatz, ${ }^{\mathrm{a}}$

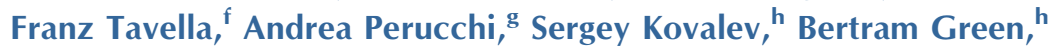 \\ Gianluca Geloni, ${ }^{,}$Takanori Tanikawa, ${ }^{,}$Mikhail Yurkov, ${ }^{a}$ Evgeny Schneidmiller, ${ }^{a}$ \\ Michael Gensch ${ }^{\mathrm{j}, \mathrm{k}}$ and Nikola Stojanovic ${ }^{\mathrm{a} *}$
}

${ }^{\mathrm{a}}$ Deutsches Elektronen-Synchrotron (DESY), Notkestrasse 85, D-22607 Hamburg, Germany, ${ }^{\mathbf{b}}$ Institute of Physics Belgrade, Pregrevica 118, 11080 Belgrade, Serbia, ${ }^{\mathrm{C} V i n c a}$ Institute of Nuclear Sciences, Belgrade, Serbia, ${ }^{\mathrm{d}}$ Center for Free-Electron Laser Science, Deutsches Elektronen-Synchrotron (DESY), Notkestrasse 85, D-22607 Hamburg, Germany, ${ }^{\mathbf{e}}$ Department of Physics, S. P. Pune University, Pune, India, '́ SLAC National Accelerator Laboratory, Menlo Park,

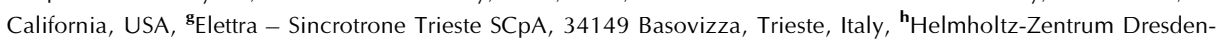
Rossendorf (HZDR), Bautzner Landstraße 400, 01328 Dresden, Germany, 'European XFEL, Holzkoppel 4, 22869 Schenefeld, Germany, 'ंGerman Aerospace Center (DLR), Institute of Optical Sensor Systems, Rutherfordstraße 2, 12489 Berlin, Germany, and 'Institute of Optics and Atomic Physics, Technical University of Berlin, Strasse des 17 Juni 135, 10623 Berlin, Germany. *Correspondence e-mail: nikola.stojanovic@desy.de

The THz beamline at FLASH, DESY, provides both tunable $(1-300 \mathrm{THz})$ narrow-bandwidth $(\sim 10 \%)$ and broad-bandwidth intense (up to $150 \mathrm{uJ}$ ) $\mathrm{THz}$ pulses delivered in $1 \mathrm{MHz}$ bursts and naturally synchronized with free-electron laser X-ray pulses. Combination of these pulses, along with the auxiliary NIR and VIS ultrashort lasers, supports a plethora of dynamic investigations in physics, material science and biology. The unique features of the FLASH THz pulses and the accelerator source, however, bring along a set of challenges in the diagnostics of their key parameters: pulse energy, spectral, temporal and spatial profiles. Here, these challenges are discussed and the pulse diagnostic tools developed at FLASH are presented. In particular, a radiometric power measurement is presented that enables the derivation of the average pulse energy within a pulse burst across the spectral range, jitter-corrected electrooptical sampling for the full spectro-temporal pulse characterization, spatial beam profiling along the beam transport line and at the sample, and a lamellar grating based Fourier transform infrared spectrometer for the on-line assessment of the average $\mathrm{THz}$ pulse spectra. Corresponding measurement results provide a comprehensive insight into the $\mathrm{THz}$ beamline capabilities.

\section{Introduction}

FLASH, the free-electron laser (FEL) in Hamburg at DESY, provides ultrafast XUV and soft X-ray radiation for users to perform pump-probe experiments. FLASH has two independent FEL undulator beamlines (Faatz et al., 2016): FLASH1 and FLASH2. Each FEL branch ends with a dedicated experimental hall that has a number of beamlines.

FLASH1 has a unique feature, a dedicated $\mathrm{THz}$ undulator installed downstream of the XUV undulators. This feature allows the generation of intense $\mathrm{THz}$ pulses by the same electron bunch that generates XUV pulses (Stojanovic \& Drescher, 2013), as shown in Fig. 1. As THz and XUV undulators are separated by empty drift space, $\mathrm{XUV}$ and $\mathrm{THz}$ pulses generated by the same electron bunch are naturally synchronized with no more than 5 fs timing jitter (Frühling et al., 2009). Furthermore, THz pulses are carrier envelope phase (CEP) stable. Downstream of the $\mathrm{THz}$ undulator, the electron beam is deflected to ground by the so-called electron beam dump magnet (hereon referred to as the dump magnet). This 
a)

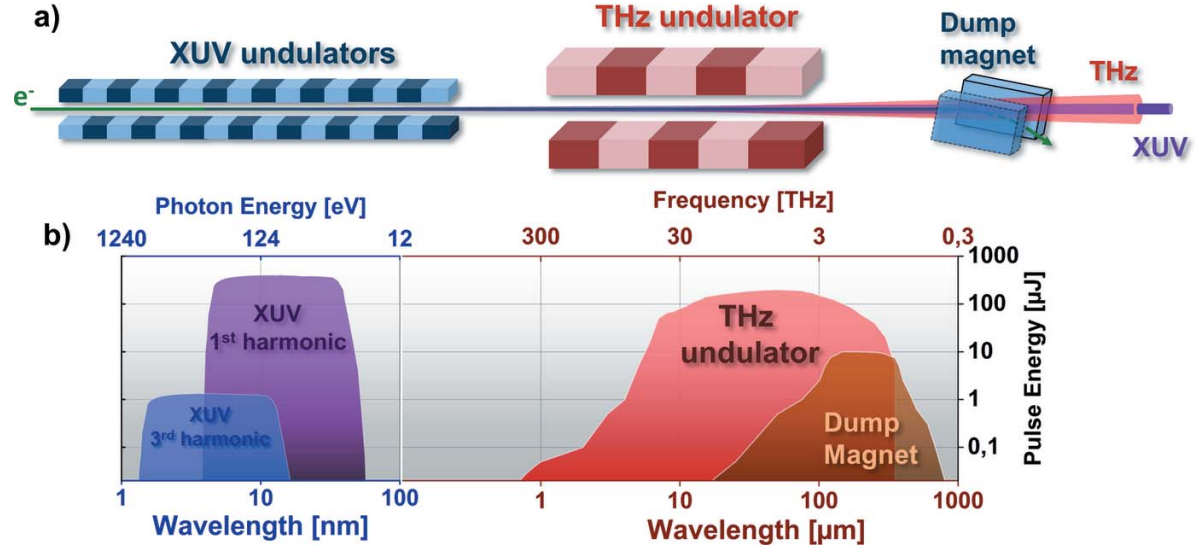

Figure 1

Scheme of the FLASH1 THz photon sources. (a) The THz undulator is located downstream of the XUV undulators, separated by free space. The electron beam dump magnet follows the $\mathrm{THz}$ undulator. (b) Representation of the pulse energies that can be obtained at FLASH1 from the XUV and $\mathrm{THz}$ sources over a wide spectral range.

stage separates the electron beam from the photon $(\mathrm{THz}$ and XUV) beams. The dump magnet on its own generates an intense $\mathrm{THz}$ transient, mainly by the edge and bending radiation process (Tavella et al., 2011; Geloni et al., 2009a,b). Only a fraction of the total bending radiation is collected in the $\mathrm{THz}$ beamline downstream (estimated to be $11.4 \%$ ), as beamline design is optimized for the radiation in the forward direction, while bending radiation is created tangentially along the bend. THz and XUV beams are separated by a large flat mirror $(210 \mathrm{~mm} \times 140 \mathrm{~mm})$ with a $10 \mathrm{~mm}$ aperture for the XUV beam (Gensch et al., 2008). Transport of the THz beam into the experimental hall over $\sim 70 \mathrm{~m}$ requires multiple collimations and this is provided by all-reflective optics. By this unique photon generation scheme, the photon spectrum of FLASH1 is extended to the long-wavelength range. As shown in Fig. 1, FLASH1 covers the XUV range from $1.4 \mathrm{~nm}$ to $52 \mathrm{~nm}$ including harmonics (Tiedtke et al., 2009), and the $\mathrm{THz}$ range from $1 \mu \mathrm{m}$ to above $300 \mu \mathrm{m}$ ( $300 \mathrm{THz}$ to $1 \mathrm{THz})$. With an independent and synchronized near-infrared (NIR) laser (Redlin et al., 2011) having a center wavelength of $800 \mathrm{~nm}$, FLASH1 can provide XUV, THz and NIR laser beams for users at the same time to study photon-matter interactions.

Based on the scheme shown in Fig. 1, there are two types of intense $\mathrm{THz}$ sources. The first is a $\mathrm{THz}$ undulator (Grimm et al., 2010) that generates tunable, linearly polarized (horizontally), narrow-bandwidth $(\Delta \lambda / \lambda=10 \%)$ radiation. The wavelength is tunable from $1 \mu \mathrm{m}$ to above $300 \mu \mathrm{m}$. The longest wavelength that can be reached depends on the electron beam energy for a given $\mathrm{THz}$ undulator period and peak field (see Fig. 2). Pulse energies delivered to the experiment can reach up to $150 \mu \mathrm{J}$, depending on the FLASH accelerator parameters (mainly the electron bunch charge and its compression).

The second source of $\mathrm{THz}$ radiation is the dump magnet that generates edge and bending radiation. The edge radiation is generated by the longitudinal acceleration of the electron beam at the interface between the free space and the dump magnet magnetic field (Tavella et al., 2011; Geloni et al., 2009a,b). This kind of radiation has a broad spectral bandwidth (quasi single-cycle temporal profile), is radially polarized and generated in the forward direction to the electron beam propagation. Electrons also generate the bending radiation along the bending arc of the dump magnet. The dump magnet vacuum chamber acceptance angle for the bending radiation is relatively small $\left(2.4^{\circ}\right)$ compared with $21^{\circ}$ of the complete bend that the electrons experience. Thus only a fraction of the bending radiation radiated in the forward radiation is collected into the $\mathrm{THz}$ beamline. Also, the bending radiation has a broad bandwidth (quasi single-cycle temporal profile) and is linearly polarized. The bending radiation is collected by the $\mathrm{THz}$ beamline mostly from the bending plane and is thus polarized mainly in the vertical direction, orthogonal to the $\mathrm{THz}$ undulator pulse polarization. Combined edge and bending dump magnet radiation can reach a pulse energy of over $10 \mu \mathrm{J}$. This radiation is generated parasitically and can be used independently from the undulator radiation.

The $\mathrm{THz}$ beamline delivers the beam to the end-station at the end of the BL3 XUV beamline in the FLASH1 experimental hall (see Fig. 3). The THz beam can be delivered to the experiment via two branches, a short one with ultra-highvacuum transport $\left(10^{-9} \mathrm{mbar}\right)$ and a long one via $\mathrm{THz}$ diagnostics hutch with high-vacuum transport $\left(10^{-7} \mathrm{mbar}\right)$. Due to the difference in optical path, the THz pulse arrives later than the XUV pulse to the end-station, $12 \mathrm{~ns}$ for the short branch and $21 \mathrm{~ns}$ for the long branch. We use two approaches to achieve temporal overlap of the XUV and THz pulses in the experiment: the first is delaying the XUV pulse by refocusing via multilayer mirrors; the second is to generate two electron bunches at the FLASH electron gun timed to achieve temporal overlap of the respective THz and XUV pulses in the experiment (Zapolnova et al., 2018).

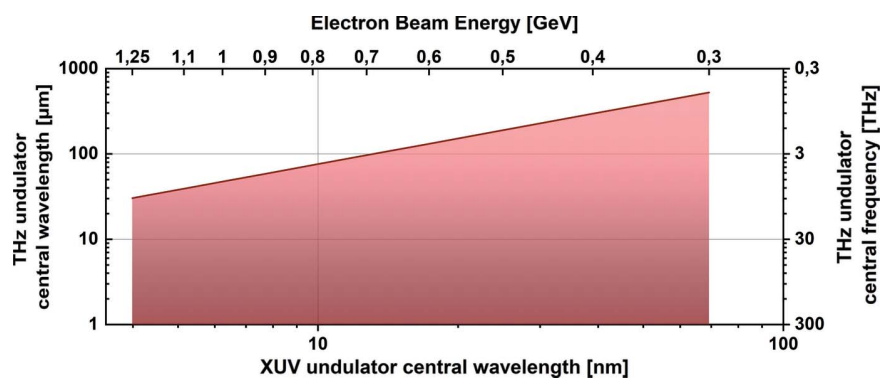

Figure 2

$\mathrm{THz}$ undulator spectral range. The shaded area represents the range where the fundamental frequency of the $\mathrm{THz}$ undulator radiation can be reached for FLASH1 as a function of the FEL XUV wavelength (lower horizontal axis) and the electron beam energy in the linac (upper horizontal axis). 


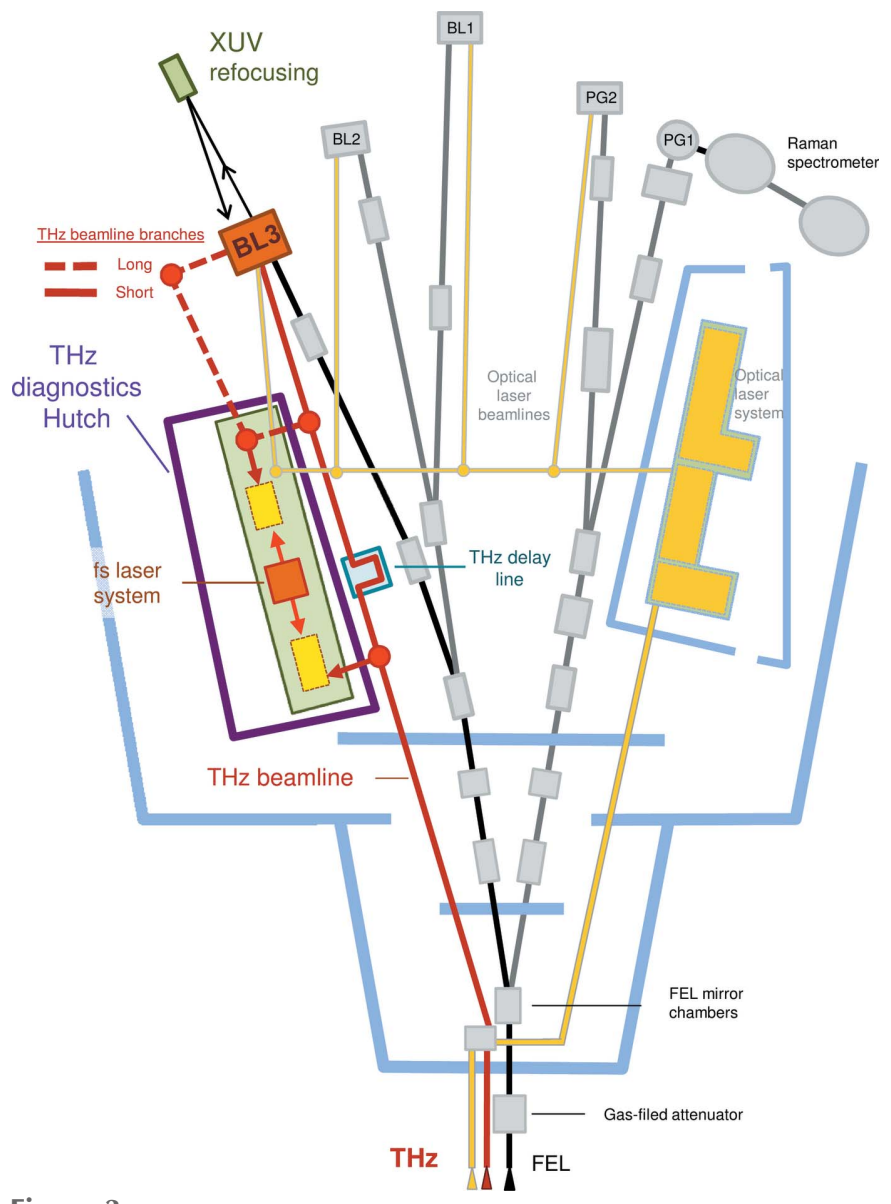

Figure 3

Scheme of the $\mathrm{THz}$ beamline in the FLASH1 experimental hall. $\mathrm{THz}$ beam is delivered to the end-station at the BL3 XUV beamline, via one of the two branches.

There are many unique applications for the CEP stable intrinsically synchronized $\mathrm{THz}$ pulses from the FLASH $\mathrm{THz}$ beamline. One important scientific area is in atomic and molecular physics. Here the $\mathrm{THz}$ field can act as a streak camera, allowing molecular reactions to be clocked and processes induced by the femtosecond-long XUV pulses from FLASH on a timescale of a few femtoseconds (Frühling et al., 2009; Schütte et al., 2012; Oelze et al., 2017; Schmid et al., 2019). An emerging new class of experiments at FLASH is the application of the strong $\mathrm{THz}$ fields for these tunable narrowband pulses in selective excitation or selective $\mathrm{THz}$ control of matter [for a description of this field see, for example, Green $e t$ al. (2016), Buzzi et al. (2018), Kampfrath et al. (2013) and Kovalev et al. (2017)]. The first experiments performed at FLASH have focused on driving the magnetization dynamics in magnetic thin films by selective phonon excitation (Radu, 2019) and on the THz control of dynamic surface processes (Waltar et al., 2018).

\section{THz diagnostics}

In a typical THz-pump/XUV-probe experiment at FLASH, determination of the properties of the driving $\mathrm{THz}$ pulse is of key importance. Based on the needs of the user experiments in past years, we have developed diagnostics tools to fully characterize the $\mathrm{THz}$ beam at the experiment. Presently, all the tools are developed in the $\mathrm{THz}$ diagnostics hutch at FLASH1 experimental hall and will be transferred to the end-station at BL3 beamline (see Fig. 3). In this paper we present tools for the full spectral, temporal and spatial characterization of the $\mathrm{THz}$ pulses and the pulse-energy measurement. Thereby we discuss some of the major challenges for diagnostics, i.e. the extremely broad $\mathrm{THz}$ spectral range of FLASH sources, $1 \mathrm{MHz}$ repetition rate in $10 \mathrm{~Hz}$ bursts and jitter to externally synchronized lasers (Azima et al., 2009; Tavella et al., 2011), that can be used for $\mathrm{THz}$ waveform characterization.

\subsection{THz power measurement}

We measure the $\mathrm{THz}$ pulse energies using a radiometer (RM3700, head RjP-735/RF, by Laser Probe). We have crossreferenced this detector to a PTB (The National Metrology Institute of Germany) traceable 3A-P-THz, by Ophir Optronics Solutions (Green et al., 2016). The radiometer detector has a cavity pyroelectric probe and it has a time constant of $1 \mathrm{~ms}$. Its temporal response prevents us from resolving individual pulses of the FLASH micro-pulses within a $1 \mathrm{MHz}$ burst. However, the detector time constant is well matched to the maximal duration of the $1 \mathrm{MHz}$ burst (with duration of $0.8 \mathrm{~ms}$, containing up to 800 pulses) defined by the FLASH accelerator. Therefore, the integration is performed over all micro-bunches in a burst, which allows for determination of the average $\mathrm{THz}$ pulse energy with good accuracy.

Fig. 4 depicts the $\mathrm{THz}$ pulse energy as a function of the central wavelength of the $\mathrm{THz}$ undulator. The total $\mathrm{THz}$ beamline transmission (from the source until the end of beamline) as a function of wavelength is depicted as well. We calculate the beamline transmission by modeling the $\mathrm{THz}$ source and the optical transport with the Synchrotron Radia-

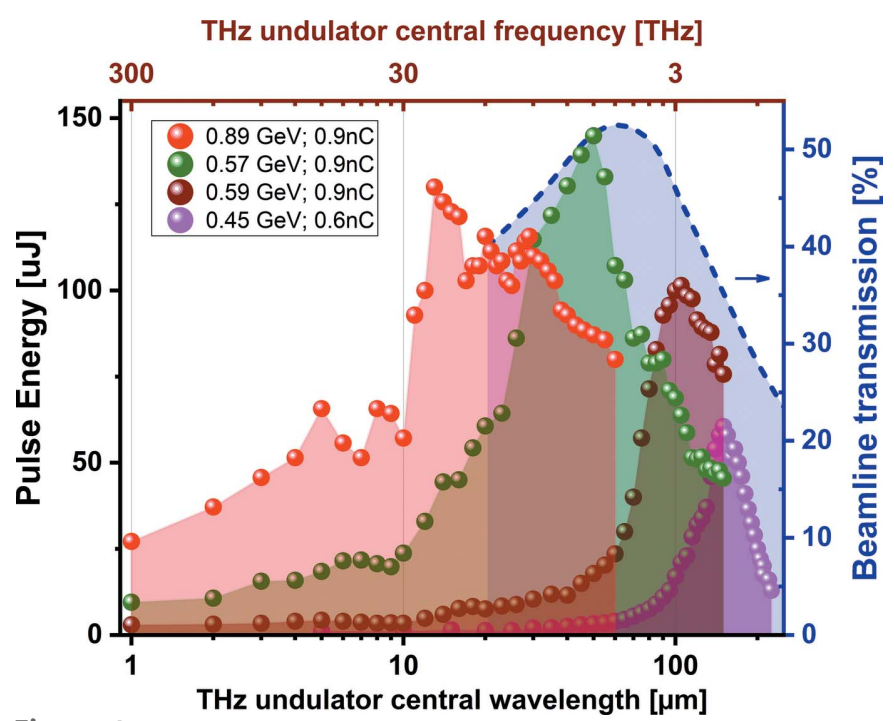

Figure 4

THz pulse energies measured at the beamline end-station for different conditions of the FLASH accelerator. The blue dashed line shows the beamline transmission, calculated using the $S R W$ software package (Chubar \& Elleaume, 1998). 
tion Workshop (SRW) software package (Chubar \& Elleaume, 1998). We also account for the Fresnel losses in the diamond window that separates the beamline from the accelerator vacuum (Gensch et al., 2008). We present examples of four measurements taken for different electron beam settings of the FLASH accelerator. Note that the abrupt end of the $\mathrm{THz}$ undulator tuning range at long wavelengths relates to the maximal wavelength that can be reached at a particular electron beam energy and is determined by the maximum field (1.2 T) inside the undulator (see also Fig. 2).

\subsection{THz temporal profile measurements}

The THz time domain spectroscopy (TDS) via electro-optic sampling (EOS) method is a well established technique for the full characterization of the THz pulse temporal structure ( $\mathrm{Wu}$ \& Zhang, 1995; Schmuttenmaer, 2004), in a broad spectral range. The electric field of the $\mathrm{THz}$ pulse changes the birefringence of the EOS crystal. This transient change is sampled by an ultrashort laser pulse. By scanning the laser pulse in time, the complete $\mathrm{THz}$ pulse shape can be reconstructed.

At an accelerator-based light source, such as FLASH, the laser system is synchronized to the master clock of the FLASH accelerator (Schulz et al., 2015). One of the main limiting factors for the use of an externally synchronized laser for EOS detection is the temporal jitter between the FEL and the laser pulses. Jitter limits the temporal resolution and subsequently the spectral bandwidth of EOS detection. We have measured the jitter of the probe laser (pulse duration $20 \mathrm{fs}$ FWHM) in the THz hutch to the FLASH THz pulses to be around $100 \mathrm{fs}$ RMS ( 200 fs peak-to-peak). To solve this, we chose to detect the THz pulses' arrival time on a single-shot basis using spectral decoding electro-optic detection (EOSD) (Jiang \& Zhang, 1998). This technique enables single-shot THz detection by imprinting the $\mathrm{THz}$ pulse electric field onto a stretched probe laser pulse, thus defining the arrival time of one with respect to the other. Spectral decoding is photon efficient (for a single-shot method) and enables the arrival time detection even with femtosecond oscillator pulses, which allows for a high-repetition-rate arrival time detection scheme that can be matched to the FLASH pulse pattern. Full EO detection of the THz pulses at FLASH then comprises two main components: arrival time monitoring by spectral decoding in combination with scanning electro-optic sampling (EOS). EOS data are sorted for their arrival time and the $\mathrm{THz}$ pulse shape is retrieved. The complete detection setup is installed in a high-vacuum chamber $\left(10^{-7} \mathrm{mbar}\right)$ to avoid measurement distortions by absorption in ambient air. We achieved a temporal resolution of the arrival time sorting of $9.7 \mathrm{fs}$ RMS. Most importantly, the EOS sampling has a bandwidth of 37$3000 \mu \mathrm{m}(0.1-8 \mathrm{THz})$ limited by the gallium phosphide (GaP) EOS crystal (100 $\mu \mathrm{m}$ thick). The setup has been developed in collaboration with the TELBE team at HZDR and details can be found in the literature (Kovalev et al., 2017; Golz, 2018).

Two typical examples of measured $\mathrm{THz}$ waveform profiles are shown in Figs. 5(a) and 5(b). The THz and the probing laser beam are overlapped and focused on the GaP EOS crystal, with beam sizes of $350 \mu \mathrm{m}$ FWHM and $70 \mu \mathrm{m}$ FWHM, respectively. The $\mathrm{THz}$ undulator was set to nominal wavelengths of $155 \mu \mathrm{m}$ and $42 \mu \mathrm{m}$ (corresponding to frequencies of $1.93 \mathrm{THz}$ and $7.1 \mathrm{TH}$, respectively). For the long-wavelength example, unfiltered and spectrally filtered pulses are presented. The wire-grid $\mathrm{THz}$ bandpass filter used has been centered at $155 \mu \mathrm{m}$ wavelength $(1.93 \mathrm{THz})$ with $15 \%$ spectral bandwidth. For the unfiltered pulse, it is interesting to observe that the electric field and high-harmonic content increase along the pulse. This indicates the change of the electron bunch form factor (Nodvick \& Saxon, 1954) inside the undulator. In the respective unfiltered $\mathrm{THz}$ pulse spectrum [see Fig. 5(c)] we clearly observe the first harmonic peaking at $169 \mu \mathrm{m}(1.77 \mathrm{THz})$ and the third harmonic at $52 \mu \mathrm{m}$ $(5.68 \mathrm{THz})$, and the baseline includes the broadband spectrum from the dump magnet radiation. As expected, the measurement with the $\mathrm{THz}$ bandpass filter shows a strong peak around $160 \mu \mathrm{m}(1.87 \mathrm{THz})$, with a small (few percent) leakage between 75 and $100 \mu \mathrm{m}$ ( 3 and $4 \mathrm{THz})$. Similarly, in the spectrum of the short-wavelength pulse (tuned to $42 \mu \mathrm{m}$ ) we observe the first harmonic peaking at $43 \mu \mathrm{m}(7 \mathrm{THz})$.
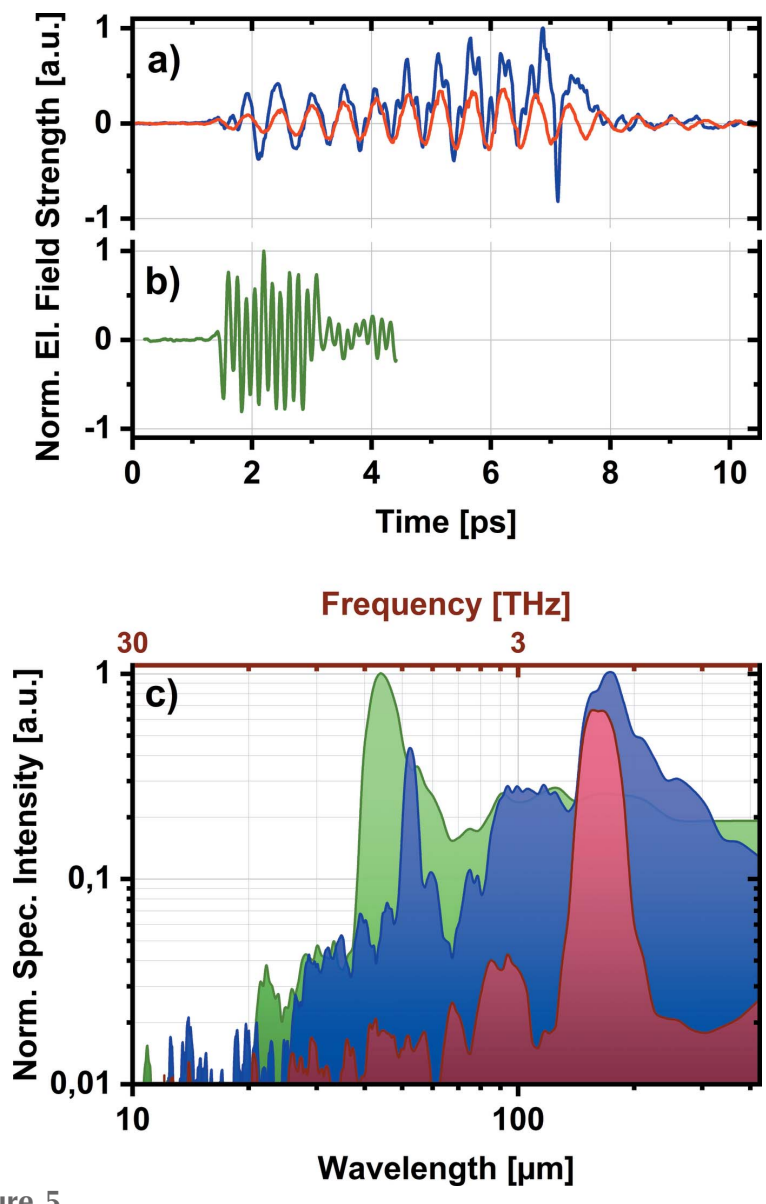

Figure 5

$\mathrm{THz}$ pulse waveforms measured by arrival-time-sorted time domain spectroscopy (TDS). (a) The THz pulse produced by the undulator set at the nominal wavelength of $155 \mu \mathrm{m}(1.93 \mathrm{THz})$. The unfiltered temporal profile is shown by the blue line and the filtered by the red line, with $155 \mu \mathrm{m}(1.93 \mathrm{THz})$ bandpass filter (15\% bandwidth). (b) The unfiltered $\mathrm{THz}$ pulse at $43 \mu \mathrm{m}(7 \mathrm{THz}$ ) (green line). (c) The corresponding $\mathrm{THz}$ pulse spectra. 
It is worth noting that the EOSD scheme can be used as a $\mathrm{THz}$ arrival-time detection tool in other experiments. A recent application evaluated the timing jitter between two electron bunches with 21 ns delay timed for temporal overlap of $\mathrm{THz}$ and XUV pulses at the experimental end-station at BL3 (Zapolnova et al., 2018).

\subsection{THz beam profile}

Knowledge of the transverse $\mathrm{THz}$ beam profile is important as it allows precise determination of the fluence (and the peak field) on the sample, as well as optimal optical design to maximize the beam transmission in the experiment. We model the $\mathrm{THz}$ source and the radiation transport using the $S R W$ software package (Chubar \& Elleaume, 1998) and observe the strong interference effects between undulator and dump magnet radiation (edge and bending radiation) at FLASH (Asgekar et al., 2014).

An example of the measured $\mathrm{THz}$ beam profile from the dump magnet, with the $\mathrm{THz}$ undulator switched off, is shown in Fig. 6(a). An example of the THz undulator beam tuned to $88 \mu \mathrm{m}(3.4 \mathrm{THz})$ is shown in Fig. 6(b). The beams have been imaged in the $\mathrm{THz}$ diagnostics hutch so that they image the plane approximately $10 \mathrm{~m}$ downstream of the radiation source. We perform 2D raster scanning of the beams. As a detector we employ an amplified pyroelectric detector (LME-501 from InfraTec). Both measurements have been performed with $30 \mu \mathrm{m}$ long-pass $\mathrm{THz}$ spectral filter. The profiles have been reproduced by $S R W$ calculation, whereby the integration of the output power was performed over the $30-300 \mu \mathrm{m}$
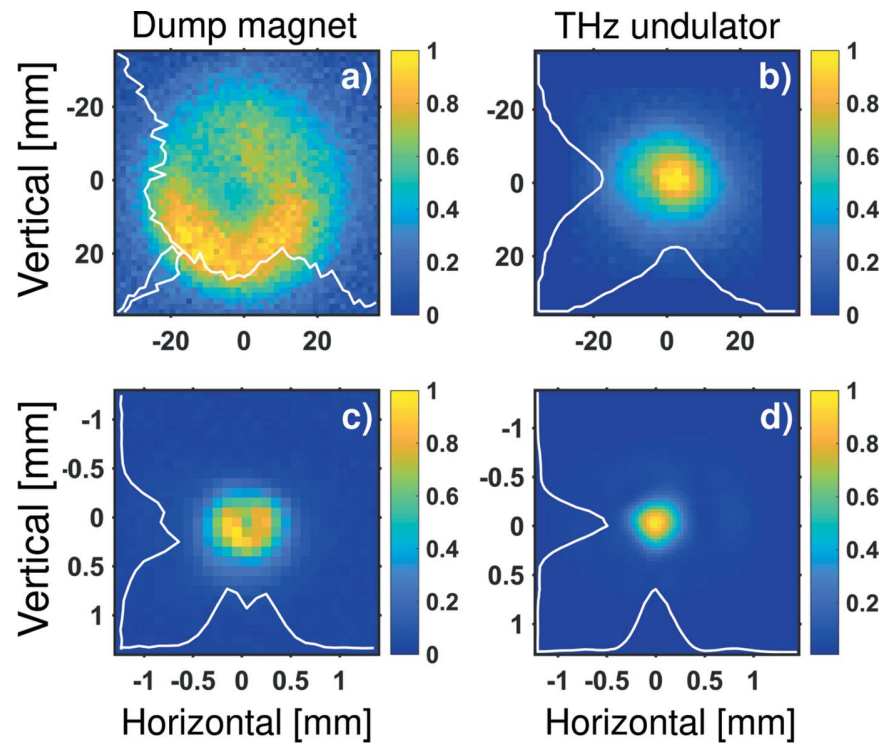

Figure 6

(Top) THz transverse beam profiles at FLASH. (a) Dump magnet (the $\mathrm{THz}$ undulator is off), and (b) THz undulator tuned to $88 \mu \mathrm{m}(3.4 \mathrm{THz})$, unfocused beam profiles measured in the $\mathrm{THz}$ hutch; beam position imaged $10 \mathrm{~m}$ from the virtual source. Note that the undulator profile is padded with zeros to fill in the same image size. (Bottom) The same beams focused. (c) Dump magnet beam (600 $\mu \mathrm{m}$ FWHM), and (d) THz undulator beam ( $350 \mu \mathrm{m}$ FWHM).
(1-10 THz) spectral range to account for the filtering, and the form factor of a 50 fs RMS long electron bunch was used.

For the dump magnet, both the measurement and calculation render a half-moon-like structure that can be explained by interference of the edge radiation with the bending radiation from the dump magnet. For the $\mathrm{THz}$ undulator beam we observe slight asymmetry in the horizontal plane which can be explained by interference of the undulator and the dump magnet radiation (Asgekar et al., 2014).

We focus both beams with an off-axis parabolic mirror of focal length $150 \mathrm{~mm}$ and measure the beam profile with a Pyrocam III camera from Ophir Photonics for the dump magnet and with a microbolometer camera for the undulator beam. The results are shown in Figs. $6(c)$ and $6(d)$, respectively. We observe a beam size of $600 \mu \mathrm{m}$ FWHM for the dump magnet and $350 \mu \mathrm{m}$ FWHM for the undulator beam.

Moreover, we follow the $\mathrm{THz}$ undulator beam profile evolution, by wavefront propagation in $S R W$. The undulator radiation wavelength is set to $160 \mu \mathrm{m}(1.87 \mathrm{THz})$. For an experimental confirmation, we measure the beam profile in the $\mathrm{THz}$ diagnostics hutch at five different positions along the beam path [see Fig. 7(a)], using the knife-edge technique. Zero
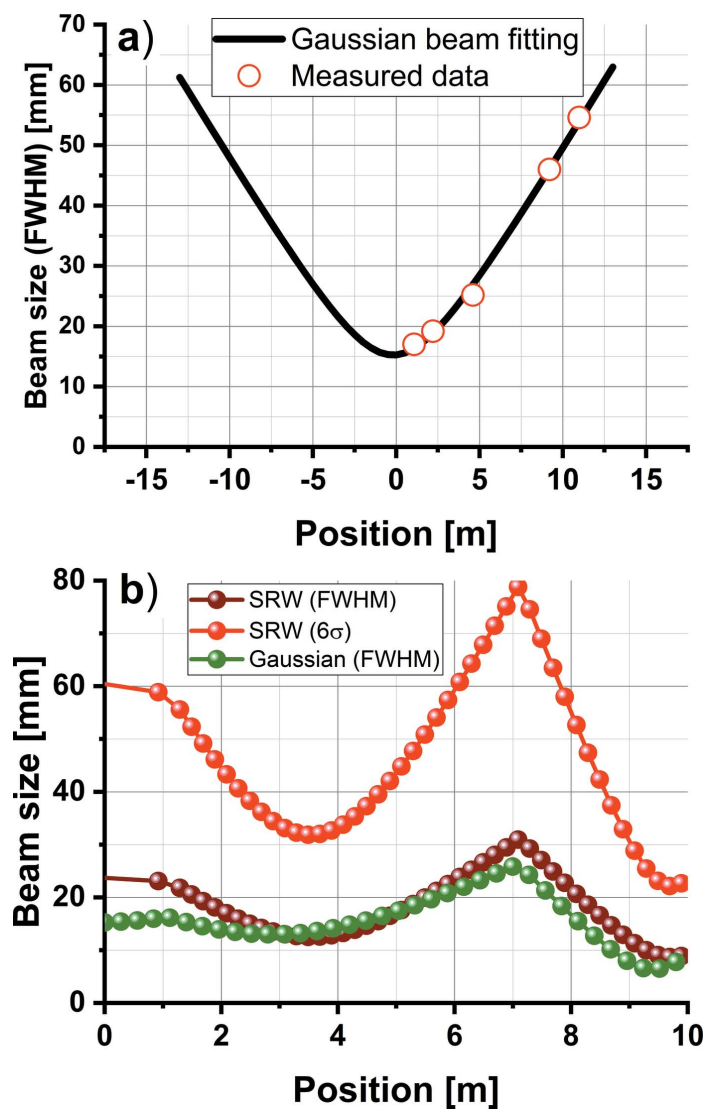

Figure 7

$\mathrm{THz}$ undulator beam size at $160 \mu \mathrm{m}(1.87 \mathrm{THz})$ and its propagation. (a) Beam size measured at five different locations along the beam path (red circles) fitted by a Gaussian beam propagation. (b) $\mathrm{THz}$ beam propagation over the $10 \mathrm{~m}$ path, with the focusing mirrors inserted at the $1 \mathrm{~m}$ and $7 \mathrm{~m}$ position marks. The THz beam was approximated by fitted Gaussian beam (FWHM) (green curve) and calculated from the source by $S R W$ (FWHM) (brown curve) and $6 \sigma$ (red curve). 
position denotes the $\mathrm{THz}$ beamline window at which the beam is extracted into the $\mathrm{THz}$ diagnostics station. We assume a Gaussian beam profile and obtain the beam parameters by fitting. The plot in Fig. 7(b) compares the beam size evolution (FWHM) calculated by $S R W$ (brown curve) with the approximated Gaussian beam propagation (ABCD matrix formalism), fitted from the measured data (green curve), for the last $10 \mathrm{~m}$ of the $\mathrm{THz}$ beamline transport. Two toroidal mirrors with focal lengths of $3.8 \mathrm{~m}$ and $1.8 \mathrm{~m}$ are inserted at the $1 \mathrm{~m}$ and $7 \mathrm{~m}$ position marks, respectively. In this particular example, the goal was to couple the $\mathrm{THz}$ beam into an experimental chamber through a $25 \mathrm{~mm}$ aperture, located at the $10 \mathrm{~m}$ position mark, with highest possible transmission. The red curve depicts the $\mathrm{THz}$ beam size at the equivalent of $6 \sigma$ (or $99.7 \%$ beam energy), and at the $10 \mathrm{~m}$ position mark we achieve the desired sub- $25 \mathrm{~mm}$ beam size. We observe a reasonable agreement between these two numerical models, with the big advantage of the Gaussian beam propagation providing very fast and efficient evaluation of the beam sizes in the optical system.

\subsection{THz spectrum measurement}

For a quick $\mathrm{THz}$ spectral characterization, when temporal pulse structure is not necessary, we have developed the variation of the Fourier transform infrared (FTIR) spectrometer based on a reflective lamellar grating. Unlike the most commonly used Michelson interferometer (based on amplitude division), the reflective lamellar grating interferometer (Richards, 1964; Bell, 1972) divides the wavefront spatially. Because of this, the reflective lamellar grating design has a key advantage: a high (close to 100\%) and smooth efficiency response (e.g. typical Fabry-Perot interferences that plague Michelson interferometers are absent). A comparison of the efficiency of the lamellar grating and the Michelson interferometer can be found in Fig. 3 of Richards (1964). As a side note, owing to a large spectral bandwidth, the lamellar gratings have found application in extreme ultraviolet (XUV) spectroscopy and metrology (Gebert et al., 2014; Usenko et al., 2017).

As shown in Fig. 8, the lamellar grating spectrometer consists of two interleaved gratings, manufactured from a $100 \mathrm{~mm}$-diameter gold-coated copper mirror. One is fixed and the other is mounted on the motorized stage responsible for introducing the optical delay between the split beams. For detection a pyroelectric detector (LME-501 from InfraTec) with a $2 \mathrm{~mm} \times 2 \mathrm{~mm}$ chip size was used.

The $\mathrm{THz}$ beam is collimated in a way that uniformly illuminates the gratings. The period of the grating $(h=2 \mathrm{~mm})$ is chosen to match the spectral range of the $\mathrm{THz}$ sources at FLASH. The long-wavelength limit (Bell, 1972) for lamellar gratings is $\lambda_{\max }<h / 2$, which is $1 \mathrm{~mm}$ (corresponding to
$0.3 \mathrm{THz}$ ) in our case. For wavelengths longer than $\lambda_{\max }$, a cavity effect starts decreasing the modulation depth of the $\mathrm{THz}$ waves, which are polarized parallel to the fringes. The high-frequency limit for this geometry is $30 \mu \mathrm{m}$ (corresponding to $10 \mathrm{THz}$ ), which is determined by diffraction theory and depends on the geometry of the device (Strong \& Vanasse, 1960; Naftaly et al., 2008; Ferhanoglu et al., 2009): $\lambda_{\min }=h s / f$, where $f=130 \mathrm{~mm}$ is the focal length of the parabolic mirror and $s=2 \mathrm{~mm}$ is the width of the exit slit of the detector (defined by the detector effective aperture).

An example of the measured interferogram and the calculated spectrum is shown in Fig. 9. The $\mathrm{THz}$ pulse was generated by the edge radiation and filtered by a $215 \mu \mathrm{m}(1.4 \mathrm{THz})$ bandpass filter. The fluctuations of the shot-to-shot THz pulse energy at FLASH can be as high as $20 \%$ RMS, depending on the FLASH accelerator settings. We split a small portion of the beam for a reference measurement that is then used to
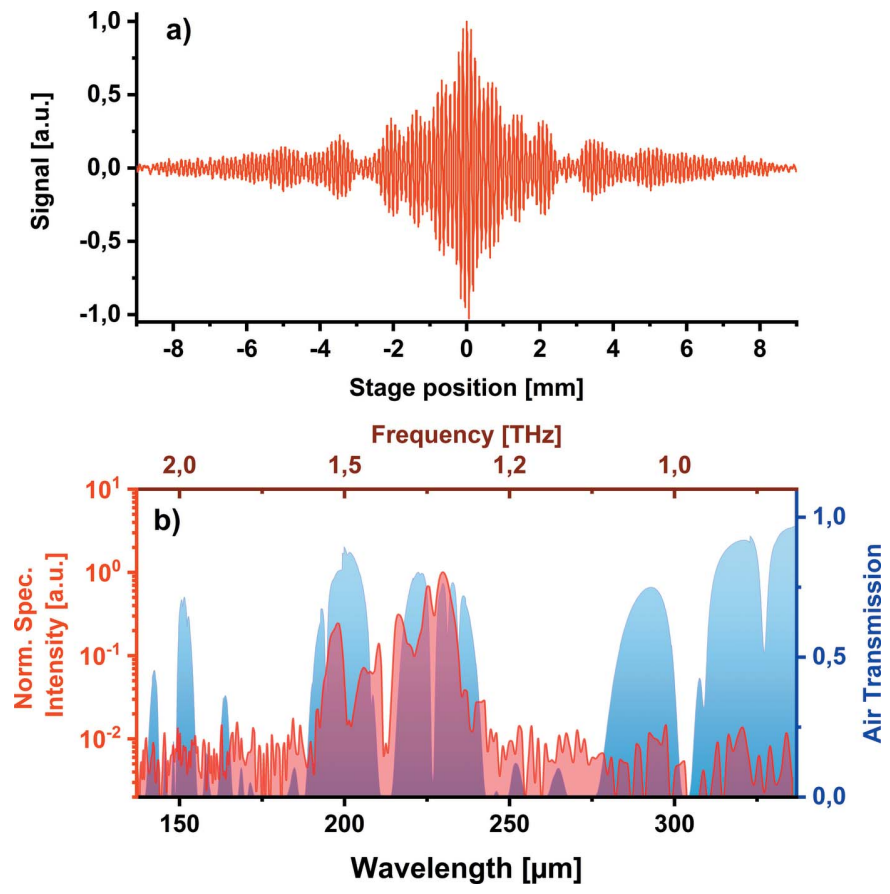

Figure 9

FTIR measurement for THz edge radiation with $215 \mu \mathrm{m}(1.4 \mathrm{THz}) \mathrm{THz}$ bandpass filter. (a) Double-sided interferogram. (b) Respective spectrum (red curve) and air transmission with water vapor (in blue) show the strong absorption lines in this spectral range. 
normalize the measured interferogram on a single-shot basis. The normalized interferogram in Fig. 9(a) was obtained during $20 \mathrm{~min}$ of scanning at $10 \mathrm{~Hz}$ repetition rate (12000 shots). Fig. $9(b)$ shows the normalized spectrum, obtained by taking a Fourier transform of the measured interferogram. We observe the spectral content as expected from the filter response with a signal-to-noise ratio exceeding 100.

Measurement has been performed in ambient air and we observe a strong modulation from the water vapor absorption lines. We are currently upgrading it to an all-in-vacuum spectrometer.

\section{Conclusion}

We have presented a range of $\mathrm{THz}$ diagnostic tools developed for THz/XUV pump-probe experiments at FLASH1, DESY. The $\mathrm{THz}$ pulse energy is an important parameter for optimization of the FLASH accelerator, and it reaches values from tens of $\mu \mathrm{J}$ up to $150 \mu \mathrm{J}$. The upgrade of a currently used radiometer to an online monitor is in progress. The $\mathrm{THz}$ temporal profile can be measured with $10 \mathrm{fs}$ timing resolution and covers the spectral range from 37 to $3000 \mu \mathrm{m}$ ( 0.1 to $8 \mathrm{THz})$. The ongoing development of this technique will explore the use of different EOS crystals to extend the measured bandwidth to shorter wavelengths, GaSe (Kübler et al., 2005) and SiC (Naftaly et al., 2016) being good candidates that should allow $\mathrm{THz}$ detection in the $10-40 \mathrm{THz}$ and $0.1-$ $15 \mathrm{THz}$ spectral windows, respectively. To fully exploit the high $\mathrm{THz}$ pulse energies at FLASH, we are upgrading the single-shot EOSD $\mathrm{THz}$ detection technique to one via tilted laser pulse front (Teo et al., 2015). This technique is free from the spectral distortions that plague EOSD (Jamison et al., 2008) and allows for the full bandwidth of the pulse to be retrieved (limited only by the EOS crystal). The measurements of the transversal $\mathrm{THz}$ beam profile are used for the design of the beam transfer line and calibration of the peak field and intensity in the experiment. A broadband FTIR spectrometer, covering the spectral range 30-1000 $\mu \mathrm{m}(0.3-$ $10 \mathrm{THz}$ ), based on a reflective lamellar grating is developed for spectral measurements. It will be permanently installed inside the $\mathrm{THz}$ beamline vacuum environment for distortionfree $\mathrm{THz}$ spectrum measurements, enabling quick and robust spectral studies [e.g. suitable for $\mathrm{THz}$ shaping by emerging THz meta-materials (Yen et al., 2004; Monticone \& Alù, 2017; Stojanović et al., 2018; Polley et al., 2018)].

\section{Funding information}

RP, EZ, AK, MR and NS acknowledge financial support from German Academic Exchange Service (DAAD Grant Numbers 57219839 and 57393513). NS acknowledge financial support from Bundesministerium für Bildung und Forschung (grant No. 05K12CH4). JP and AP were supported by the 'Stephenson Distinguished Visitor Programme' at DESY in Hamburg (Germany). JP, MR and AK acknowledge support from the Ministry of Education, Science and Technological Development of Serbia, under Grant No. III45010 and OI
171038 and bilateral projects 451-03-01732/2017-09/6 and 45103-01038/2015-09/1. MG and BG acknowledge support from the European Cluster of Advanced Laser Light Sources (EUCALL) project which has received funding from the European Union's Horizon 2020 research and innovation program under grant agreement No 654220. VA acknowledges support from the Alexander von Humboldt Foundation, Germany.

\section{References}

Asgekar, V., Geloni, G., Kocharyan, V., Stojanovic, N., Michel, P. \& Gensch, M. (2014). Infrared Phys. Technol. 64, 26-32.

Azima, A., Düsterer, S., Radcliffe, P., Redlin, H., Stojanovic, N., Li, W., Schlarb, H., Feldhaus, J., Cubaynes, D., Meyer, M., Dardis, J., Hayden, P., Hough, P., Richardson, V., Kennedy, E. T. \& Costello, J. T. (2009). Appl. Phys. Lett. 94, 144102.

Bell, R. J. (1972). Introductory Fourier Transform Infrared Spectroscopy. New York: Academic Press.

Buzzi, M., Först, M., Mankowsky, R. \& Cavalleri, A. (2018). Nat. Rev. Mater. 3, 299-311.

Chubar, O. \& Elleaume, P. (1998). Proceedings of the Sixth European Particle Accelerator Conference (EPAC'98), 22-26 June 1998, Stockholm, Sweden, pp. 1177-1179.

Faatz, B., Plönjes, E., Ackermann, S., Agababyan, A., Asgekar, V., Ayvazyan, V., Baark, S., Baboi, N., Balandin, V., Bargen, N., Bican, Y., Bilani, O., Bödewadt, J., Böhnert, M., Böspflug, R., Bonfigt, S., Bolz, H., Borges, F., Borkenhagen, O., Brachmanski, M., Braune, M., Brinkmann, A., Brovko, O., Bruns, T., Castro, P., Chen, J., Czwalinna, M. K., Damker, H., Decking, W., Degenhardt, M., Delfs, A., Delfs, T., Deng, H., Dressel, M., Duhme, H., Düsterer, S., Eckoldt, H., Eislage, A., Felber, M., Feldhaus, J., Gessler, P., Gibau, M., Golubeva, N., Golz, T., Gonschior, J., Grebentsov, A., Grecki, M., Grün, C., Grunewald, S., Hacker, K., Hänisch, L., Hage, A., Hans, T., Hass, E., Hauberg, A., Hensler, O., Hesse, M., Heuck, K., Hidvegi, A., Holz, M., Honkavaara, K., Höppner, H., Ignatenko, A., Jäger, J., Jastrow, U., Kammering, R., Karstensen, S., Kaukher, A., Kay, H., Keil, B., Klose, K., Kocharyan, V., Köpke, M., Körfer, M., Kook, W., Krause, B., Krebs, O., Kreis, S., Krivan, F., Kuhlmann, J., Kuhlmann, M., Kube, G., Laarmann, T., Lechner, C., Lederer, S., Leuschner, A., Liebertz, D., Liebing, J., Liedtke, A., Lilje, L., Limberg, T., Lipka, D., Liu, B., Lorbeer, B., Ludwig, K., Mahn, H., Marinkovic, G., Martens, C., Marutzky, F., Maslocv, M., Meissner, D., Mildner, N., Miltchev, V., Molnar, S., Mross, D., Müller, F., Neumann, R., Neumann, P., Nölle, D., Obier, F., Pelzer, M., Peters, H., Petersen, K., Petrosyan, A., Petrosyan, G., Petrosyan, L., Petrosyan, V., Petrov, A., Pfeiffer, S., Piotrowski, A., Pisarov, Z., Plath, T., Pototzki, P., Prandolini, M. J., Prenting, J., Priebe, G., Racky, B., Ramm, T., Rehlich, K., Riedel, R., Roggli, M., Röhling, M., Rönsch-Schulenburg, J., Rossbach, J., Rybnikov, V., Schäfer, J., Schaffran, J., Schlarb, H., Schlesselmann, G., Schlösser, M., Schmid, P., Schmidt, C., Schmidt-Föhre, F., Schmitz, M., Schneidmiller, E., Schöps, A., Scholz, M., Schreiber, S., Schütt, K., Schütz, U., SchulteSchrepping, H., Schulz, M., Shabunov, A., Smirnov, P., Sombrowski, E., Sorokin, A., Sparr, B., Spengler, J., Staack, M., Stadler, M., Stechmann, C., Steffen, B., Stojanovic, N., Sychev, V., Syresin, E., Tanikawa, T., Tavella, F., Tesch, N., Tiedtke, K., Tischer, M., Treusch, R., Tripathi, S., Vagin, P., Vetrov, P., Vilcins, S., Vogt, M., Wagner, A. Z., Wamsat, T., Weddig, H., Weichert, G., Weigelt, H., Wentowski, N., Wiebers, C., Wilksen, T., Willner, A., Wittenburg, K., Wohlenberg, T., Wortmann, J., Wurth, W., Yurkov, M., Zagorodnov, I. \& Zemella, J. (2016). New J. Phys. 18, 062002.

Ferhanoglu, O., Seren, H. R., Lüttjohann, S. \& Urey, H. (2009). Opt. Express, 17, 21289-21301. 
Frühling, U., Wieland, M., Gensch, M., Gebert, T., Schütte, B., Krikunova, M., Kalms, R., Budzyn, F., Grimm, O., Rossbach, L., Plönjes, E. \& Drescher, M. (2009). Nat. Photon. 3, 523.

Gebert, T., Rompotis, D., Wieland, M., Karimi, F., Azima, A. \& Drescher, M. (2014). New J. Phys. 16, 073047.

Geloni, G., Kocharyan, V., Saldin, E., Schneidmiller, E. \& Yurkov, M. (2009a). Nucl. Instrum. Methods Phys. Res. A, 605, 409-429.

Geloni, G., Kocharyan, V., Saldin, E., Schneidmiller, E. \& Yurkov, M. (2009b). Nucl. Instrum. Methods Phys. Res. A, 607, 470-487.

Gensch, M., Bittner, L., Chesnov, A., Delsim-Hashemi, H., Drescher, M., Faatz, B., Feldhaus, J., Fruehling, U., Geloni, G., Gerth, C., Grimm, O., Hahn, U., Hesse, M., Kapitzki, S., Kocharyan, V., Kozlov, O., Matyushevsky, E., Morozov, N., Petrov, D., Ploenjes, E., Roehling, M., Rossbach, J., Saldin, E. L., Schmidt, B., Schmueser, P., Schneidmiller, E. A., Syresin, E., Willner, A. \& Yurkov, M. V. (2008). Infrared Phys. Technol. 51, 423-425.

Golz, T. (2018). PhD thesis, Hamburg University, Germany.

Green, B., Kovalev, S., Asgekar, V., Geloni, G., Lehnert, U., Golz, T., Kuntzsch, M., Bauer, C., Hauser, J., Voigtlaender, J., Wustmann, B., Koesterke, I., Schwarz, M., Freitag, M., Arnold, A., Teichert, J., Justus, M., Seidel, W., Ilgner, C., Awari, N., Nicoletti, D., Kaiser, S., Laplace, Y., Rajasekaran, S., Zhang, L., Winnerl, S., Schneider, H., Schay, G., Lorincz, I., Rauscher, A. A., Radu, I., Mährlein, S., Kim, T. H., Lee, J. S., Kampfrath, T., Wall, S., Heberle, J., MalnasiCsizmadia, A., Steiger, A., Müller, A. S., Helm, M., Schramm, U., Cowan, T., Michel, P., Cavalleri, A., Fisher, A. S., Stojanovic, N. \& Gensch, M. (2016). Sci. Rep. 6, 22256.

Grimm, O., Morozov, N., Chesnov, A., Holler, Y., Matushevsky, E., Petrov, D., Rossbach, J., Syresin, E. \& Yurkov, M. (2010). Nucl. Instrum. Methods Phys. Res. A, 615, 105-113.

Jamison, S., Gillespie, W. \& Phillips, P. (2008). Signal, 1, 2.

Jiang, Z. \& Zhang, X.-C. (1998). Appl. Phys. Lett. 72, 1945-1947.

Kampfrath, T., Tanaka, K. \& Nelson, K. A. (2013). Nat. Photon. 7, 680.

Kovalev, S., Green, B., Golz, T., Maehrlein, S., Stojanovic, N., Fisher, A. S., Kampfrath, T. \& Gensch, M. (2017). Struct. Dyn. 4, 024301.

Kübler, C., Huber, R. \& Leitenstorfer, A. (2005). Semicond. Sci. Technol. 20, S128-S133.

Monticone, F. \& Alù, A. (2017). Rep. Prog. Phys. 80, 036401.

Naftaly, M., Dean, P., Miles, R. E., Fletcher, J. R. \& Malcoci, A. (2008). Quantum Electron. 14, 443-448.

Naftaly, M., Molloy, J., Magnusson, B., Andreev, Y. \& Lanskii, G. (2016). Opt. Express, 24, 2590-2595.

Nodvick, J. S. \& Saxon, D. S. (1954). Phys. Rev. 96, 180-184.

Oelze, T., Schütte, B., Müller, M., Müller, J. P., Wieland, M., Frühling, U., Drescher, M., Al-Shemmary, A., Golz, T., Stojanovic, N. \& Krikunova, M. (2017). Sci. Rep. 7, 40736.

Polley, D., Hagström, N. Z., Schmising, C. K., Eisebitt, S. \& Bonetti, S. (2018). J. Phys. B At. Mol. Opt. Phys. 51, 224001.
Radu, I. (2019). In preparation.

Redlin, H., Al-Shemmary, A., Azima, A., Stojanovic, N., Tavella, F., Will, I. \& Düsterer, S. (2011). Nucl. Instrum. Methods Phys. Res. A, 635, S88-S93.

Richards, P. L. (1964). J. Opt. Soc. Am. 54, 1474-1484.

Schmid, G., Schnorr, K., Augustin, S., Meister, S., Lindenblatt, H., Trost, F., Liu, Y., Stojanovic, N., Al-Shemmary, A., Golz, T., Treusch, R., Gensch, M., Kübel, M., Foucar, L., Rudenko, A., Ullrich, J., Schröter, C. D., Pfeifer, T. \& Moshammer, R. (2019). Phys. Rev. Lett. 122, 073001.

Schmuttenmaer, C. A. (2004). Chem. Rev. 104, 1759-1779.

Schulz, S., Grguraš, I., Behrens, C., Bromberger, H., Costello, J., Czwalinna, M., Felber, M., Hoffmann, M., Ilchen, M., Liu, H., Mazza, T., Meyer, M., Pfeiffer, S., Prędki, P., Schefer, S., Schmidt, C., Wegner, U., Schlarb, H. \& Cavalieri, A. L. (2015). Nat. Commun. 6, 5938.

Schütte, B., Bauch, S., Frühling, U., Wieland, M., Gensch, M., Plönjes, E., Gaumnitz, T., Azima, A., Bonitz, M. \& Drescher, M. (2012). Phys. Rev. Lett. 108, 253003.

Stojanović, D. B., Beličev, P. P., Gligorić, G. \& Hadžievski, L. (2018). J. Phys. D Appl. Phys. 51, 045106.

Stojanovic, N. \& Drescher, M. (2013). J. Phys. B At. Mol. Opt. Phys. 46, 192001.

Strong, J. \& Vanasse, G. A. (1960). J. Opt. Soc. Am. 50, 113118.

Tavella, F., Stojanovic, N., Geloni, G. \& Gensch, M. (2011). Nat. Photon., 5, 162.

Teo, S. M., Ofori-Okai, B. K., Werley, C. A. \& Nelson, K. A. (2015). Rev. Sci. Instrum. 86, 051301.

Tiedtke, K., Azima, A., von Bargen, N., Bittner, L., Bonfigt, S., Düsterer, S., Faatz, B., Frühling, U., Gensch, M., Gerth, C., Guerassimova, N., Hahn, U., Hans, T., Hesse, M., Honkavaar, K., Jastrow, U., Juranic, P., Kapitzki, S., Keitel, B., Kracht, T., Kuhlmann, M., Li, W. B., Martins, M., Núñez, T., Plönjes, E., Redlin, H., Saldin, E. L., Schneidmiller, E. A., Schneider, J. R., Schreiber, S., Stojanovic, N., Tavella, F., Toleikis, S., Treusch, R., Weigelt, H., Wellhöfer, M., Wabnitz, H., Yurkov, M. V. \& Feldhaus, J. (2009). New J. Phys. 11, 023029.

Usenko, S., Przystawik, A., Jakob, M. A., Lazzarino, L. L., Brenner, G., Toleikis, S., Haunhorst, C., Kip, D. \& Laarmann, T. (2017). Nat. Commun. 8, 15626.

Waltar, K., Haase, J., Lucchini, M., van Bokhoven, J. A., Hengsberger, M., Osterwalder, J. \& Castiglioni, L. (2018). Opt. Express, 26, 83648374.

Wu, Q. \& Zhang, X. (1995). Appl. Phys. Lett. 67, 3523-3525.

Yen, T.-J., Padilla, W., Fang, N., Vier, D., Smith, D., Pendry, J., Basov, D. \& Zhang, X. (2004). Science, 303, 1494-1496.

Zapolnova, E., Golz, T., Pan, R., Klose, K., Schreiber, S. \& Stojanovic, N. (2018). J. Synchrotron Rad. 25, 39-43. 